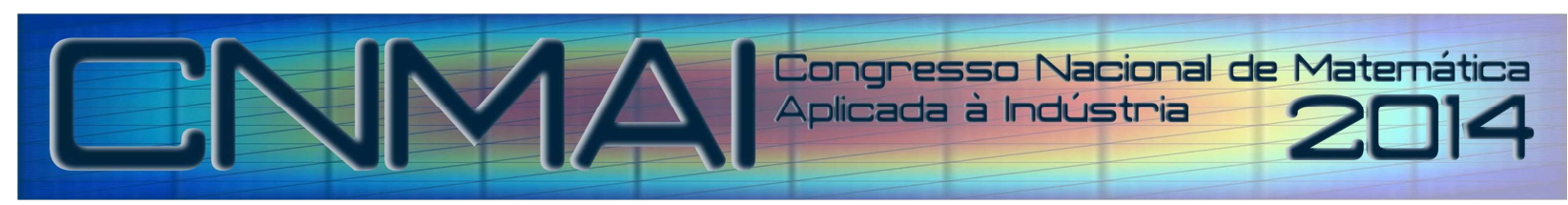

18 a 21 de novembro de 2014, Caldas Novas - Goiás

\title{
SIMULAÇÃO COMPUTACIONAL DE UM MODELO MAGNETOSTÁTICO USANDO O MÉTODO DE ELEMENTOS FINITOS APLICADO A UM TORÓIDE
}

\author{
Juliana Almansa Malagoli, juliana.malagoni@gmail.com ${ }^{1}$ \\ José Roberto Camacho, jrcamacho@ufu.br ${ }^{1}$ \\ Mauricio Valencia Ferreira da Luz, mauricio.luz@ufsc.br ${ }^{2}$ \\ Jacson Hudson Inácio Ferreira, Jacson@iftm.edu.br ${ }^{1}$
}

\begin{abstract}
${ }^{1}$ Universidade Federal de Uberlândia - Faculdade de Engenharia Elétrica.
Av.: João Naves de Ávila, no 2121, Bairro: Santa Mônica, CEP: 38.408-100, Uberlândia-MG, Brasil.

${ }^{2}$ Universidade Federal de Santa Catarina - Departamento de Engenharia Elétrica.

Campus Universitário Reitor João David Ferreira Lima, Bairro: Trindade, CEP: 88.040-970, Florianópolis-SC, Brasil.

Resumo: O projeto de toróide, forma geométrica do núcleo redondo e semelhante a um anel e cuja função base é concentrar as linhas de força do campo magnético, configura-se como um interessante tema de pesquisa, já que está diretamente relacionado aos custos de transformadores. Este trabalho tem como objetivo desenvolver um modelo matemático fundamentado nas Equações de Maxwell e nas leis de comportamento dos materiais através da discretização dos campos para a análise do problema de magnetostática para um toróide. Neste cenário, a metodologia proposta permite determinar parâmetros em dispositivos eletromagnéticos ainda na fase do projeto, de forma que, simulações computacionais contribuam para uma análise antecipada do funcionamento do dispositivo antes da construção do protótipo. Para o desenvolvimento deste trabalho optou-se por utilizar três ferramentas computacionais de uso público, a saber, i) o Gmsh, gerador de malhas de elementos finitos em 3D, ii) o GetDP, ambiente para tratamento de problemas discretos modelados através de equações diferenciais e iii) o FEMM, simulador de problemas em 2D de elementos finitos. Nas simulações apresentaram-se as induções magnéticas e os campos magnéticos de um toróide bidimensional e tridimensional utilizando o método de elementos finitos. Os resultados mostram que a metodologia proposta configurou-se como uma interessante estratégia para a finalidade a que se destina esse trabalho.
\end{abstract}

Palavras-chave: Método de Elementos Finitos, Modelo Magnetostático, Gmsh/GetDP, FEMM, Toróide.

\section{INTRODUÇÃO}

Muitos problemas físicos são desenvolvidos matematicamente na forma de equações diferenciais parciais e ordinárias. Para geometrias simples é possível obter uma solução analítica exata utilizando métodos algébricos. Todavia, para a maioria das aplicações, com geometrias mais complexas, a obtenção da solução analítica é praticamente impossível. Assim, esses problemas exigem a aplicação de métodos numéricos (Malagoni, 2012).

O Método dos Elementos Finitos (MEF) consiste em um método numérico aproximado para análise de diversos fenômenos físicos que ocorrem em meios contínuos, e que são descritos através de equações diferenciais parciais, com determinadas condições de contorno (problemas de valor de contorno) e com condições iniciais (para problemas variáveis no tempo). O MEF é bastante genérico e pode ser aplicado na solução de inúmeros problemas da engenharia. A ideia principal do Método dos Elementos Finitos consiste em se dividir o domínio (meio contínuo) do problema em sub-regiões de geometria simples (formato triangular, quadrilateral, cúbico, etc.).

Etapas para aplicação do Método dos Elementos Finitos (Ferreira da Luz, 2003; Arend, 2009 e Malagoni, 2012):

A. Pré-Processamento:

- Definir o problema e o domínio;

- Discretizar ou dividir o domínio em elementos; 
B. Processamento:

- Obter as equações dos elementos;

- Escolher a função de aproximação;

- Montar ou colocar as equações dos elementos juntas;

- Adicionar as condições iniciais e de contorno;

- Resolver o sistema linear (ou não linear);

C. Pós-Processamento:

- Apresentar os resultados ou visualizar os gráficos;

- Determinar as variáveis secundárias.

Mesmo que o MEF tenha sido originalmente desenvolvido para a análise de sistemas estruturais, o mesmo tem sido utilizado também nos domínios da mecânica dos sólidos, dos fluidos, eletromagnetismo, transmissão de calor e de massa, dentre outros (Malagoni, 2012 e Arend, 2009). Além de sua adequação à programação em computadores digitais, a sua eficiência e flexibilidade, o MEF tem hoje uma grande difusão tanto no meio acadêmico como no industrial, estando disponível em grande número os softwares existentes no mercado: livres (FEMM e GMSH/GETDP) e comerciais (ANSYS, COMSOL, FLUX 3D, etc.).

Em se tratando do projeto de sistemas de engenharia, objetiva-se desenvolver um modelo matemático fundamentado nas equações de Maxwell e nas leis de comportamento dos materiais através da discretização dos campos para a análise do problema de magnetostática aplicado a um toróide. Deseja-se com o projeto deste sistema apresentar as induções magnéticas e os campos magnéticos de um toróide bidimensional e tridimensional utilizando o método de elementos finitos.

As próximas seções deste artigo estarão organizadas da seguinte maneira: na seção 2 será abordado o modelo matemático magnetostático aplicado ao toróide. Na seção 3 será mostrado as simulações e resultados obtidos para apresentar as induções magnéticas e os campos magnéticos do toróide. Por fim, na seção 4 estarão as considerações finais.

\section{MODELAGEM MAGNETOSTÁTICO}

O modelo magnetostático consiste no estudo dos fenômenos magnéticos em regime estacionário (Dular, 1996; Dular, Legros, Nicolet, 1998; Ferreira da Luz, 2003 e Malagoni, 2012). Considerando o domínio de estudo $\Omega$, de fronteira $\Gamma$, o modelo magnetostático é caracterizado pelas equações de Maxwell, pelas condições de contorno e pelas leis de comportamento, que são:

$$
\begin{gathered}
\operatorname{rot} \boldsymbol{H}=\boldsymbol{J} \\
\operatorname{div} \boldsymbol{B}=0 \\
\boldsymbol{B}=\mu \boldsymbol{H} \\
\boldsymbol{n} \times\left.\boldsymbol{H}\right|_{\Gamma_{H}}=0 \\
\left.\boldsymbol{n} \cdot \boldsymbol{B}\right|_{\Gamma_{B}}=0 \\
\Gamma=\Gamma_{H} \cup \Gamma_{B}
\end{gathered}
$$

Onde $\boldsymbol{H}$ é o campo magnético $(A / m), \boldsymbol{J}$ é a densidade de corrente de condução $\left(A / m^{2}\right), \boldsymbol{B}$ é a indução magnética $(T), \mu$ é a permeabilidade magnética e $\boldsymbol{n}$ é o vetor normal à superfície.

As restrições globais definidas são relativas ao fluxo magnético $\Phi_{i}$ e a força magnetomotriz $F m m_{i}$, cuja relação define o inverso de uma relutância $\mathfrak{R}$.

$$
\begin{gathered}
\int_{\Gamma_{i}} \boldsymbol{n} \cdot \boldsymbol{B} d s=\Phi_{i} \\
\int_{\gamma_{i}} \boldsymbol{H} \cdot d \boldsymbol{l}=F m m_{i} \\
\frac{1}{\mathfrak{R}}=\frac{\Phi_{i}}{F m m_{i}}
\end{gathered}
$$


Essa forma inicial do problema constitui sua formulação forte. Neste trabalho são apresentadas as utilizações do vetor potencial magnético.

Na Formulação do Vetor Potencial Magnético o divergente da indução magnética é nulo como observado na Eq. (2). Isso decorre diretamente do fato de não existirem cargas magnéticas isoladas da mesma maneira que as cargas elétricas positivas ou negativas. Uma segunda forma de enunciar essa propriedade fundamental da conservação de fluxo da indução magnética é dizer que a indução é solenoidal. A condição div $\boldsymbol{B}=0$ permite então definir uma função vetor potencial magnético, $\boldsymbol{a}$, tal que:

$$
\boldsymbol{B}=\operatorname{rot} \boldsymbol{a}, \quad \operatorname{com} \boldsymbol{a} \times \boldsymbol{n}=0 \mathrm{em} \Gamma_{B}
$$

Substituindo-se esta expressão nas Eq. (1) e (3), obtém-se:

$$
\operatorname{rot}\left(\frac{1}{\mu} \operatorname{rot} \boldsymbol{a}\right)=\boldsymbol{J}
$$

Na prática, a unicidade da solução é obtida com a ajuda das condições de contorno. Como visto anteriormente, uma maneira de resolver o conjunto de equações de Maxwell do modelo magnetostático é usando a definição de vetor potencial magnético a. Assim, a equação resultante é a Eq. (11). Esta forma inicial do problema é o que tem sido considerado até agora e constitui sua formulação forte.

A forma fraca da Eq. (11) é obtida da seguinte maneira: suponha que a função $\boldsymbol{a}$ seja uma função aproximada, de maneira que a Eq. (11) se torne:

$$
R=\operatorname{rot}\left(\frac{1}{\mu} \operatorname{rot} \boldsymbol{a}\right)-\boldsymbol{J}
$$

onde $R$ é um resíduo, visto que a função $\boldsymbol{a}$ não é exata.

O objetivo é fazer com que o resíduo tenha a tendência de se anular ou ainda que na média ponderada o resíduo seja zero. Matematicamente, isso se escreve da seguinte forma (Reece, Preston, 2000),

$$
\int_{\Omega} R W d \Omega=0 \quad \text { ou }(R, W)_{\Omega}=0
$$

Onde $W$ é a função de ponderação. Esse método de resolver a Eq. (13) é conhecido como Método dos Resíduos Ponderados (Dular, 1996; Geuzaine, 2001; Ferreira da Luz, 2003). Substituindo a Eq. (12) em (13), tem-se:

$$
\left(\operatorname{rot}\left(\frac{1}{\mu} \operatorname{rot} \boldsymbol{a}\right)-\boldsymbol{J}, W\right)_{\Omega}=0
$$

Fazendo $W=\boldsymbol{a}^{\prime}$ e aplicando a fórmula de Green do tipo rot-rot, onde $u=\boldsymbol{H}=\frac{1}{\mu}$ rot $\boldsymbol{a}$ e $v=\boldsymbol{a}^{\prime}$, tem-se:

$$
\left(\frac{1}{\mu} \operatorname{rot} \boldsymbol{a}, \operatorname{rot} \boldsymbol{a}^{\prime}\right)_{\Omega}-\left(\boldsymbol{J}, \boldsymbol{a}^{\prime}\right)_{\Omega}+<\boldsymbol{n} \times \boldsymbol{H}, \boldsymbol{a}^{\prime}>_{\Gamma}=0, \quad \forall \boldsymbol{a}^{\prime} \in F_{u}^{1}
$$

Onde $F_{u}^{1}=\left\{\boldsymbol{a} \in \boldsymbol{L}^{2}(\Omega) ; \operatorname{rot} \boldsymbol{a} \in \boldsymbol{L}^{2}(\Omega), \boldsymbol{n} \times\left.\boldsymbol{a}\right|_{\Gamma_{u}}=0\right\}$.

O terceiro termo a esquerda da Eq. (15) pode ser dividido como sendo:

$$
<\boldsymbol{n} \times \boldsymbol{H}, \boldsymbol{a}^{\prime}>_{\Gamma}=<\boldsymbol{n} \times \boldsymbol{H}, \boldsymbol{a}^{\prime}>_{\Gamma_{H}}+<\boldsymbol{n} \times \boldsymbol{H}, \boldsymbol{a}^{\prime}>_{\Gamma_{B}}
$$

O primeiro termo da Eq. (16) do lado direito é uma condição de contorno natural, pode ser nula devido $\boldsymbol{n} \times$ $\left.\boldsymbol{H}\right|_{\Gamma_{H}}=0$ ou pode associar às quantidades globais do tipo circulação, como por exemplo, a força magnetomotriz. $\mathrm{O}$ segundo termo do lado direito é nulo, pois $\left.\boldsymbol{n} \cdot \boldsymbol{B}\right|_{\Gamma_{B}}=0$.

Desse modo, tomando a integral de superfície sobre $\Gamma_{H}$ nula, a Eq. (15) se torna,

$$
\left(\frac{1}{\mu} \operatorname{rot} \boldsymbol{a}, \operatorname{rot} \boldsymbol{a}^{\prime}\right)_{\Omega}=\left(\boldsymbol{J}, \boldsymbol{a}^{\prime}\right)_{\Omega}, \quad \forall \boldsymbol{a}^{\prime} \in F_{u}^{1}
$$

Outra forma de escrever a Eq. (17) é dada por: 


$$
\int_{\Omega}^{1} \frac{1}{\mu} \operatorname{rot} \boldsymbol{a} \cdot \operatorname{rot} \boldsymbol{a}^{\prime} d \Omega-\int_{\Omega} \boldsymbol{J} \cdot \boldsymbol{a}^{\prime} d \Omega=0, \quad \forall \boldsymbol{a}^{\prime} \in F_{u}^{1}
$$

As Eq. (17) e (18) representam a formulação magnetostática fraca em vetor potencial magnético $\boldsymbol{a}$.

Na seção 3 será mostrado as simulações e resultados obtidos para apresentar as induções magnéticas e os campos magnéticos do toróide.

\section{SIMULAÇÕES E RESULTADOS}

Nesta seção é apresentado um toróide de $N$ espiras e dimensões tais que, a indução magnética $B$ em seu interior possa ser aproximada como uniforme, é tal que $R c$ é o raio da circunferência que passa até o centro da espira e o raio até a extremidade interna do toróide é $R e=R c-r$, onde $r$ é o raio da espira como mostra a Fig. 1.

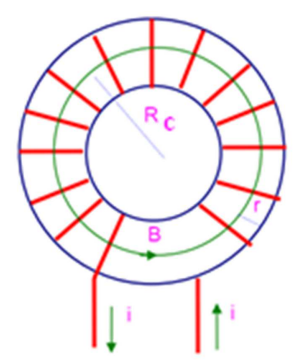

Figura 1. Modelo do Toróide estudado.

O toróide possui núcleo de ar, 2000 (espiras), corrente de $15(A)$, o raio que passa até o centro da espira é $0,13(\mathrm{~m})$ e o raio da extremidade interna do toróide é $0,10(\mathrm{~m})$. Analisa-se a indução magnética ou densidade de fluxo magnético $B(T)$ e o campo magnético $H(A / m)$ no centro e na extremidade interna do toróide.

O método de elementos finitos é aplicado para um toróide 3D (três dimensões). O modelo matemático magnetostático foi desenvolvido no item 2 deste artigo com as condições de contorno do domínio estudado.

Para comparar os resultados da simulação em 3D no Gmsh/GetDP com os valores dos cálculos analíticos, fez-se o modelo em 2D (duas dimensões) no software livre FEMM (Finite Element Method Magnetics).

A Figura 2 (a) mostra o topo do toróide em duas dimensões no software FEMM, ou seja, o modelo e as malhas geradas do mesmo. Já a Fig. 2 (b) mostra a vista frontal do toróide em três dimensões no software Gmsh/GetDP, ou seja, somente o modelo é observado sem as malhas.

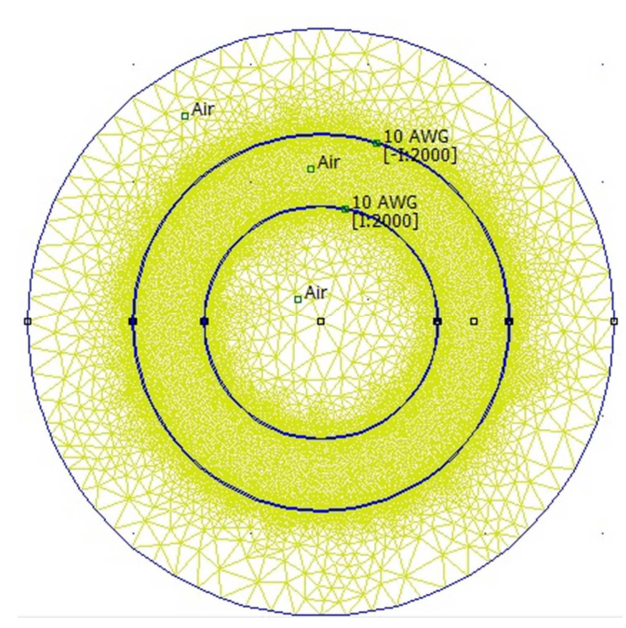

(a)

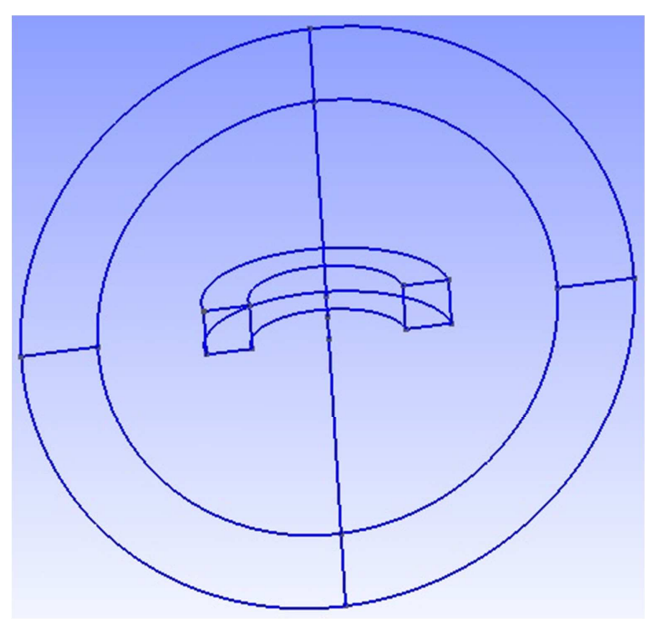

(b)

Figura 2. Modelo do Toróide com malha (a) no FEMM em 2D e sem malha (b) no Gmsh/GetDP em 3D.

A principal contribuição deste trabalho é o desenvolvimento de um programa para simular o modelo estudado magnetostático utilizando método de elementos finitos. O software FEMM é de uso simples para analisar o toróide, já o software Gmsh/GetDP é necessário programar para gerar os resultados de análises do método numérico aplicado. A Tabela 1 mostra uma parte do programa desenvolvido, por exemplo, descrevendo a Eq. (18) que representa a formulação magnetostática fraca em vetor potencial magnético $\boldsymbol{a}$. 
Tabela 1. Exemplo de uma parte do programa desenvolvido, no caso as formulações.

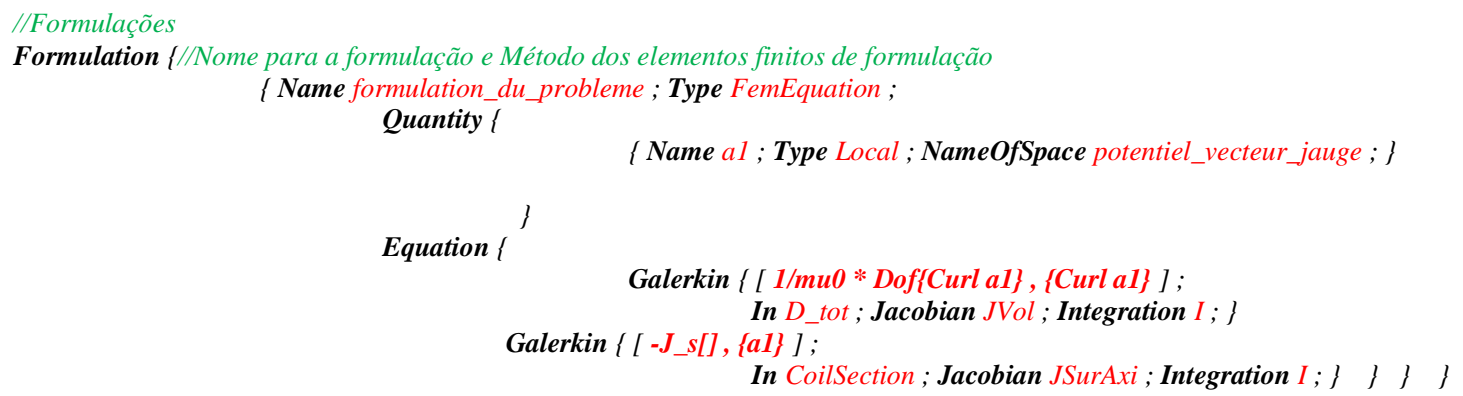

Na Tabela 1, os comentários estão de verde, os escritos de preto com negrito são estruturas do programa GetDP para escrever as formulações, as chaves e os colchetes são representados por preto sem negrito, os escritos de vermelho sem negrito são nomes criados e por último a Eq. (18) está descrita de vermelho com negrito.

A Figura 3 (a) e (b) mostra a distribuição da indução magnética $B(T)$ no FEMM e no Gmsh/GetDP, repectivamente. Observa-se que os valores são próximos $B=0,0591(T)$ no FEMM e $B=0,0592(T)$ no Gmsh/GetDP.

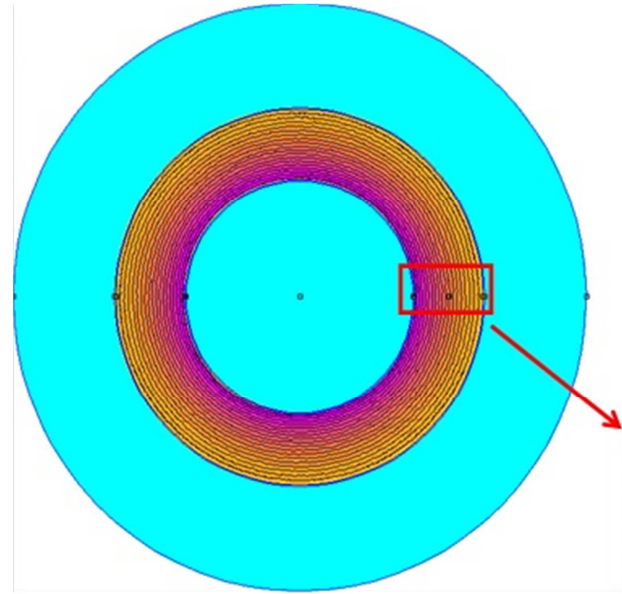

(a)

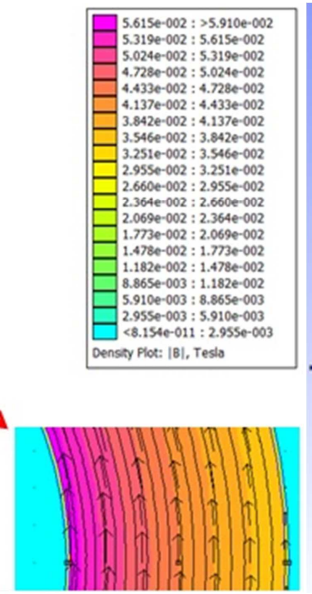

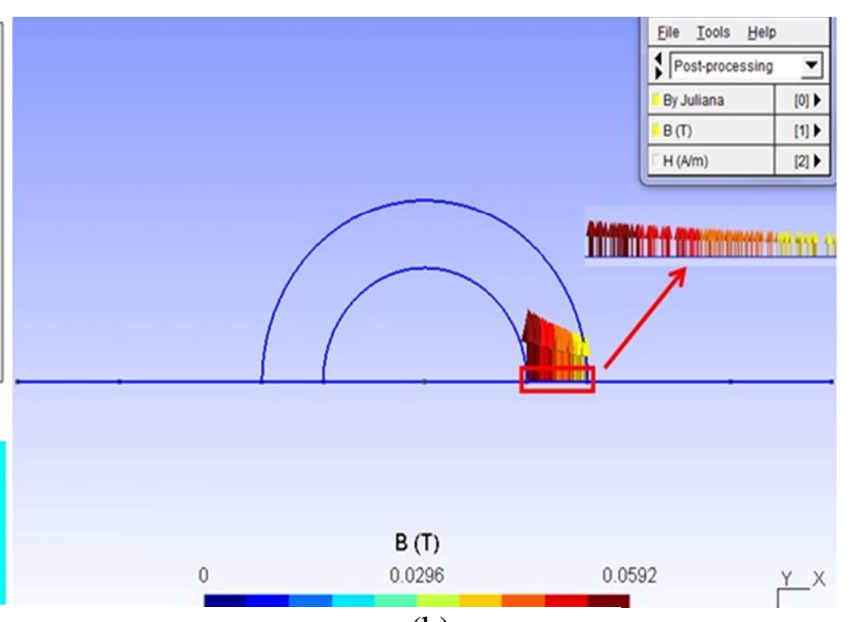

(b)

Figura 3. Distribuição da indução magnética $B(T)$ (a) no FEMM em 2D e (b) no Gmsh/GetDP em 3D.

Já a Fig. 4 (a) e (b) mostra a distribuição do campo magnético $H$ no FEMM e no Gmsh/GetDP, repectivamente. Observa-se que os valores são próximos $H=47.087(\mathrm{~A} / \mathrm{m})$ no FEMM e $H=47.100(\mathrm{~A} / \mathrm{m})$ no Gmsh/GetDP.

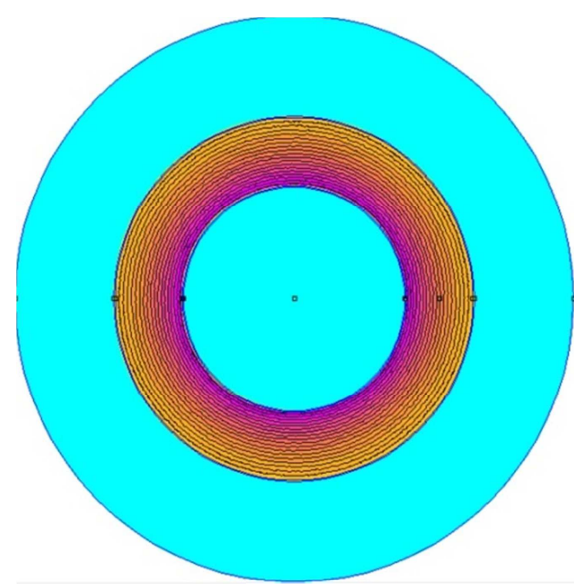

(b)
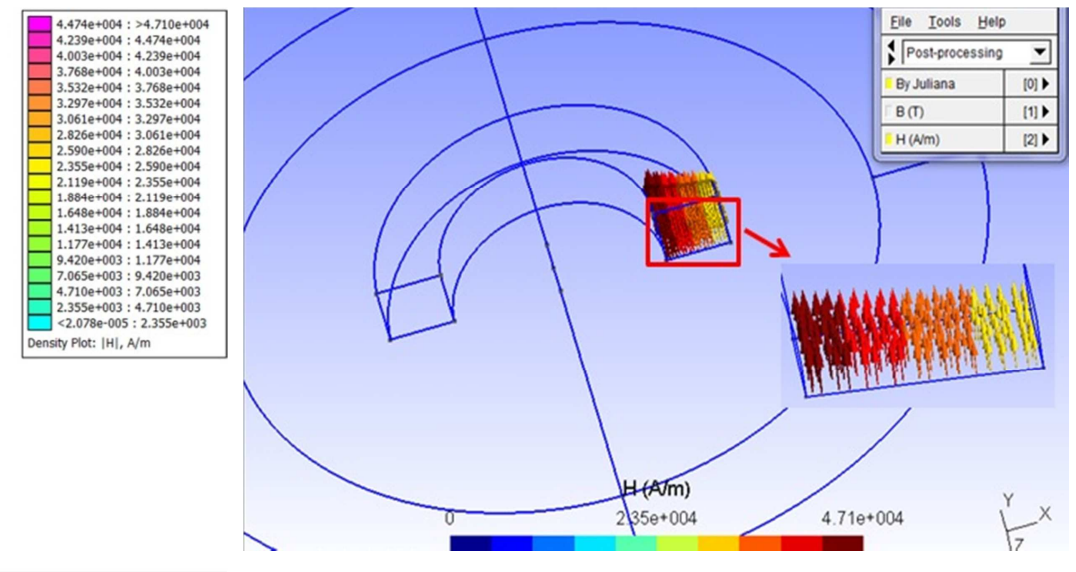

(b)

Figura 4. Distribuição do campo magnético $H(A / m)$ (a) no FEMM em $2 D$ e (b) no Gmsh/GetDP em 3D.

Comparou-se os resultados simulados nos softwares FEMM e Gmsh/GetDP com cálculos analíticos. Então, fizeram-se os cálculos analíticos da indução magnética $B$ e do campo magnético $H$ do toróide. 
Utilizando a Lei de Ampère, a indução magnética $B$ no centro e na extremidade interna do toróide são dadas por (Guimarães, 2010):

$$
B c=\frac{\mu_{0} N I}{2 \pi R c}
$$

Onde $B c$ é a indução magnética no centro do toróide $(T) ; \mu_{0}$ é a permeabilidade magnética do vácuo (de valor numérico $\left.4 \pi * 10^{-7} \mathrm{H} / \mathrm{m}\right) ; N$ é o número de espiras; $I$ é a corrente $(A) ; R c$ é o raio que passa até o centro do toróide $(\mathrm{m})$.

$$
B e=\frac{\mu_{0} N I}{2 \pi R e}
$$

Onde $B e$ é a indução magnética na extremidade interna do toróide $(T)$; Re é o raio até a extremidade interna do toróide $(m)$.

A lei de comportamento do material (ou relação constitutiva), que estabelece a relação entre campo magnético e o meio em que estão inseridos é dada pela seguinte equação (Guimarães, 2010):

$$
H c=\frac{B c}{\mu_{0}}
$$

Onde $H c$ é o campo magnético no centro do toróide $(A / m)$.

$$
H e=\frac{B e}{\mu_{0}}
$$

Onde He é o campo magnético na extremidade interna do toróide $(A / m)$.

A Tab. 2 apresenta os resultados analíticos e simulados nos software FEMM e Gmsh/GetDP, respectivamente. Além disso, analisaram-se os campos magnéticos e a indução magnética no centro e na extremidade do toróide estudado.

Tabela 2. Resultados e Comparações do modelo magnetostático no FEMM em 2D e no Gmsh/GetDP em 3D.

\begin{tabular}{lccc}
\hline Resultados Analisados & Cálculo Analítico & FEMM & Gmsh/GetDP \\
\hline Indução Magnética - $B c(T)$ & 0,0462 & 0,0463 & 0,0465 \\
\hline Indução Magnética - Be $(T)$ & 0,0600 & 0,0591 & 0,0592 \\
\hline Campo Magnético - Hc $(A / m)$ & $36,765 * 10^{3}$ & $36,822 * 10^{3}$ & $36,900 * 10^{3}$ \\
\hline Campo Magnético - He $(A / m)$ & $47,746 * 10^{3}$ & $47,087 * 10^{3}$ & $47,100^{*} 10^{3}$ \\
\hline
\end{tabular}

A Tab. 3 apresenta os erros percentuais dos resultados dos cálculos analíticos e simulados do modelo magnetostático.

Tabela 3. Erros dos resultados do modelo magnetostático no Gmsh/GetDP em 3D e no FEMM em 2D.

\begin{tabular}{lccc}
\hline \multicolumn{1}{c}{ Erros } & Analítico-Gmsh/GetDP $(\%)$ & Analítico-FEMM $(\%)$ & Gmsh/GetDP-FEMM (\%) \\
\hline Indução Magnética - $B c(T)$ & 0,649 & 0,216 & 0,430 \\
\hline Indução Magnética - $B e(T)$ & 1,333 & 1,500 & 0,169 \\
\hline Campo Magnético - $H c(A / m)$ & 0,375 & 0,155 & 0,211 \\
\hline Campo Magnético - $H e(A / m)$ & 1,353 & 1,400 & 0,028 \\
\hline
\end{tabular}

Observa-se erros pequenos nos resultados do FEMM e do Gmsh/GetDP em comparação aos cálculos analíticos. Além disso, os cálculos realizados em cada software são diferentes. E o modelo feito no FEMM em duas dimensões é escalar (representado por mapa de cores), enquanto no Gmsh/GetDP em três dimensões é vetorial (representado por setas tridimensionais). Pode-se concluir também que nas simulações o campo magnético de um toroíde está inteiramente confinado ao espaço no interior das espiras (o campo é zero fora do toroíde). 


\section{CONCLUSÕES}

O estudo realizado no presente trabalho teve como objetivo a modelagem de um toróide através de uma metodologia computacional e de modelos matemáticos, levando em conta as equações de Maxwell, as leis de comportamento dos materiais e as condições de contorno. Além da discretização dos campos pelo método de elementos finitos utilizando o método de Galerkin, destinados à análise de problemas de eletromagnetismo.

$\mathrm{Na}$ maioria das vezes medições reais são de difícil execução e sujeitas a erros de medidas. O MEF permite determinar parâmetros em dispositivos eletromagnéticos ainda na fase do projeto, de forma que, simulações computacionais, tais como as realizadas neste trabalho, contribuem para uma análise antecipada do funcionamento do dispositivo antes da construção do protótipo. Essa vantagem é mais evidente em dispositivos onde não é possível a construção de protótipos, como é o caso de máquinas elétricas e transformadores de potências elevadas.

De uma maneira geral, este trabalho contribuiu com alguns estudos e análises que vão dar subsídios a trabalhos futuros mais complexos como, por exemplo, a modelagem e simulação de máquinas elétricas. Destaca-se a confiabilidade dos softwares livres Gmsh/GetDP para a determinação de variáveis magnetostáticas e ressalta-se que a formulação magnetostática fraca em vetor potencial aplicada nesse trabalho foi adequada para se obter os parâmetros desejados.

\section{AGRADECIMENTOS}

A autora Juliana Almansa Malagoli agradece à CAPES pelos recursos destinados ao desenvolvimento deste trabalho de pesquisa.

\section{REFERÊNCIAS}

Arend, E. R. 2009. Estudo de Aterramento em Baixas Frequências Usando a Formulação Eletrocinética Associada ao Método de Elementos Finitos. Dissertação de Mestrado, Centro Tecnológico, Universidade Federal de Santa Catarina.

Dular, P. 1996. Modélisation du champ magnétique et des courants induits dans des systèmes tridimensionnels non linéaires. Tese de Doutorado, Universidade de Liège, Bélgica.

Dular, P., Legros, W., Nicolet, A. 1998. Coupling of Local and Global Quantities in Various Finite Element Formulations and its Application to Electrostatics, Magnetostatics and Magnetodynamics. IEEE Transactions on Magnetics, vol. 34, № 5, Setembro.

Ferreira da Luz, M. V. 2003. Desenvolvimento de um Software para Cálculo de Campos Eletromagnéticos 3D Utilizando Elementos de Aresta, Levando em Conta o Movimento e o Circuito de Alimentação. Tese de Doutorado, Centro Tecnológico, Universidade Federal de Santa Catarina.

Geuzaine, C. 2001. High order hybrid finite element schemes for Maxwell's equations taking thin structures and global quantities into account. Tese de Doutorado, Université de Liège, outubro, Bélgica.

Guimarães, G. C. 2010, Apostila de Conceitos Teóricos e Exercícios Propostos de Eletromagnetismo. Faculdade de Engenharia Elétrica. Universidade Federal de Uberlândia.

Malagoni, J. A. 2012. Os Elementos Finitos no Estudo de Eletromagnetismo Utilizando os Software Gmsh/GetDP. Dissertação de Mestrado, Faculdade de Engenharia Elétrica, Universidade Federal de Uberlândia.

Reece, A. B. J.; Preston, T. W. 2000. Finite Element Methods in Electrical Power Engineering. Oxford University Press.

\section{RESPONSABILIDADE AUTORAL}

Os autores são os únicos responsáveis pelo conteúdo deste trabalho. 


\title{
COMPUTER SIMULATION OF A MODEL MAGNETOSTATIC USING FINITE ELEMENT METHOD APPLIED TO A TOROID
}

\author{
Juliana Almansa Malagoli, juliana.malagoni@gmail.com ${ }^{1}$ \\ José Roberto Camacho,jrcamacho@ufu.br ${ }^{1}$ \\ Mauricio Valencia Ferreira da Luz, mauricio.luz@ufsc.br ${ }^{2}$ \\ Jacson Hudson Inácio Ferreira, Jacson@iftm.edu.br ${ }^{1}$
}

${ }^{1}$ Federal University of Uberlândia - Faculty of Electrical Engineering.

Av.: João Naves de Ávila, no 2121, Bairro: Santa Mônica, CEP: 38.408-100, Uberlândia-MG, Brazil.

${ }^{2}$ Federal University of Santa Catarina - Department of Electrical Engineering.

Campus Universitário Reitor João David Ferreira Lima, Bairro: Trindade, CEP: 88.040-970, Florianópolis-SC, Brazil.

\begin{abstract}
The toroid design, geometric shape of the round core and the like a ring and whose basic function is to concentrate the lines of force the magnetic field, appears as an interesting research topic, since it is directly related to the costs of transformers. This paper aims develop a mathematical model based on Maxwell's equations and the laws of behavior material by discretization fields for the analysis of magnetostatic problem for a toroid. In the scenario, the proposed methodology allows to determine parameters in electromagnetic devices still in the design phase, so that computer simulations contribute for an early analysis of the device operation before building the prototype. To develop this paper chose three software tools for public use, namely, i) Gmsh, generator of finite element mesh in 3D, ii) GetDP, environment for the treatment of discrete problems modeled by differential equations and iii) FEMM, 2D simulator problems of finite elements. In the simulations presented is the magnetic induction and magnetic fields a twodimensional and three-dimensional toroid using the finite element method. The results show that the proposed methodology is configured as an interesting strategy for the purpose it is intended that paper.
\end{abstract}

Keywords: Finite Element Method, Magnetostatic Model, Gmsh/GetDP, FEMM, Toroid. 